\title{
REFLECTING POWER OF MONEL METAL, STELLITE, AND ZINC
}

By W. W. Coblentz

The object of this paper is to place on record the reflective properties of several alloys, about which inquiries have been received as to their applicability for mirrors.

The apparatus and methods were essentially the same as used in previous work of this type. ${ }^{1}$ For observations in the visible spectrum a lens spectrometer and light flint-glass prism were used. The source of radiation was a Nernst glower which was focused upon the slit by means of an achromatic lens $6 \mathrm{~cm}$ in diameter and $\mathrm{I} 8 \mathrm{~cm}$ focal length.

The spectral intensities were measured by means of a vacuum thermopile of bismuth and silver, and an ironclad Thomson galvanometer. The galvanometer deflections were never less than $50 \mathrm{~mm}$; a high precision was therefore possible in the measurements.

The reflecting power of the sample under test was determined by comparison with a silvered mirror placed at the entrance slit of the spectrometer as illustrated in Fig. I of Scientific Paper No. 308. In order to determine the absolute reflecting power the observed data were multiplied by the spectral reflecting power of the silver mirror given in Table $I$, which is about the average of the values of various observers. In view of the great variations in the polish of samples of metals, this method of determining the reflecting power has been found to give results which are as accurate as a direct determination of the reflectivity (Scientific Paper No. 308). 
TABLE 1.-Reflecting Power of Metals

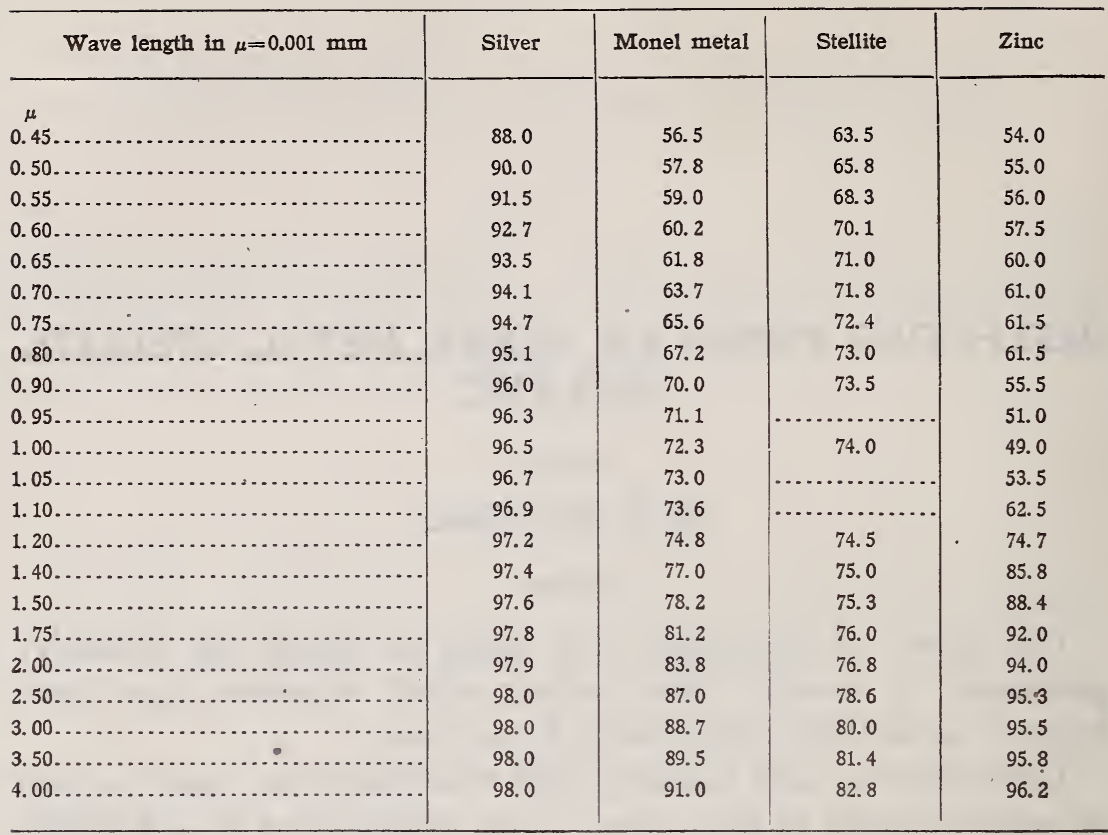

For measurements in the infra-red, a mirror spectrometer and a quartz prism were used. The source of radiation was a gasfilled tungsten lamp. The samples were placed at the exit slit, an image of which was focused upon the thermopile by means of a mirror as shown in Fig. 2 of Scientific Paper No. 338. In this manner it was easy to cause all the radiation, including some of the diffusely reflected rays, reflected from the sample to fall upon the thermopile receiver, which was $2 \mathrm{~mm}$ wide. One can therefore use samples which are not perfectly flat.

MONEL METAL.-This is a natural alloy, found in Canada, consisting of 68 to 70 per cent nickel, I.5 per cent of iron, and the remainder of copper. It takes a high polish, does not tarnish readily, and hence is useful for mirrors. The sample examined was refined material sawed from a $\operatorname{rod}^{2} 32 \mathrm{~mm}$ in diameter. Its surface was optically flat and had a high polish.

The observed reflecting power, in the visible spectrum, is from 2 to 4 per cent lower than that of nickel, which is to be expected in view of the fact that copper and iron have a lower reflecting power than nickel in this region.

${ }^{2}$ Kindly supplied by Dr. P. D. Merica, The International Nickel Co., Bayonne, N. J. 
Beyond $\mathrm{I} \mu$ in the infra-red, the reflecting power of monel metal is closely the same as that of pure nickel. Apparently the copper constituent, which has a higher reflectivity than nickel in the infra-red, is not present in a sufficient amount to affect the reflectivity.

STELLITE.-Data on the reflectivity of this sample, No. 2, of stellite were published in Scientific Paper No. 342 (19r9). A fluorite prism having been used at that time, the dispersion was small in the visible spectrum. It was therefore desirable to repeat the measurements using the present apparatus, in which both the intensity of the light and dispersion were higher, and hence a higher accuracy was attainable. For example, on remounting the mirror, the observations were repeated to 0.5 per cent. The new data are given in Table $\mathrm{I}$. In the region of $0.55 \mu$ to $0.65 \mu$ they are about I per cent lower than previously published.

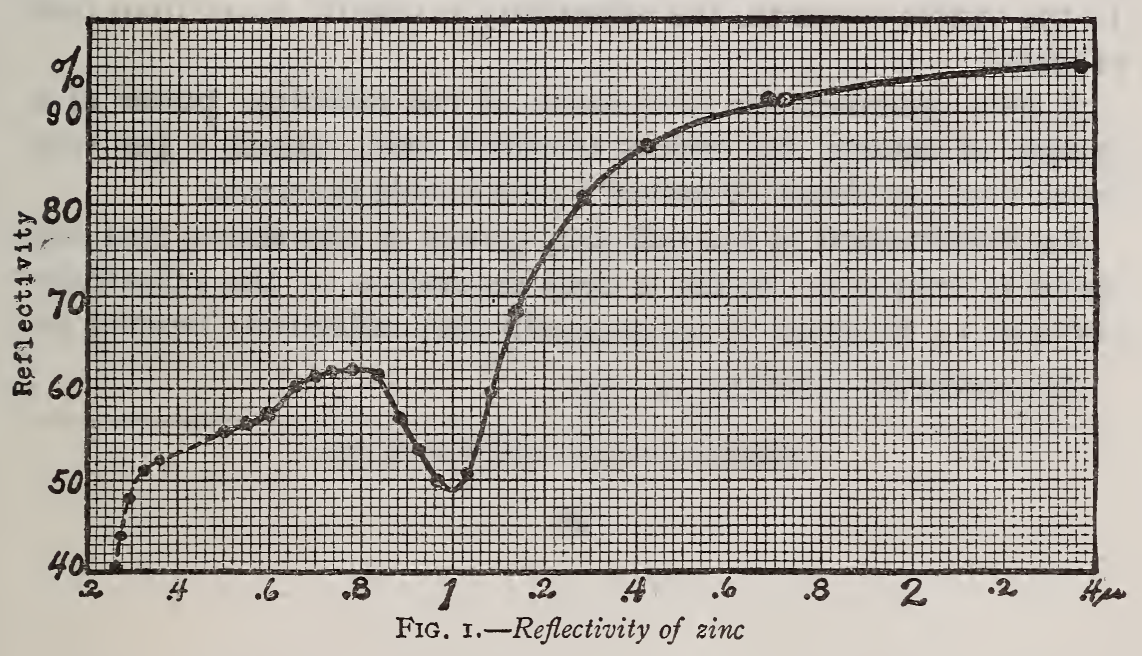

ZINc.-The infra-red reflecting power of zinc was determined some time ago. ${ }^{3}$ The reflectivity curve rises very abruptly at $I \mu$, which indicates the presence of an absorption band in this part of the spectrum. In connection with an investigation of the reflecting power of zinc and magnesium alloys of aluminum, ${ }^{4}$ it was found that the zinc alloys showed a reflectivity minimum at $I \mu$. An examination of the above-mentioned sample of zinc (surface poorly polished) showed this same reflectivity minimum. This is so unusual that it was deemed desirable to repeat the work. using a newly polished surface.

${ }^{3}$ Publication No. 65, Carnegie Inst. of Washington, 1906; B. S. Scientific Papers, No. 45 ; 1907.

$\checkmark$ B. S. Scientific Papers, No. 363; 1920. 
The reflectivity curve of zinc is shown in Fig. I. The dotted part of the curve in the ultra violet is plotted from data published by Hulburt. ${ }^{5}$ The point marked with a circle $(\odot)$ at $1.7 \mu$ is taken from the first paper, in which the observations were obtained with a fluorite prism and a vacuum bolometer. The old data are in excellent agreement with those just obtained on the newly polished surface, and using a different method of observation.

The spectral reflectivity of zinc is unique in having a deep depression at $\mathrm{I} \mu$, followed by an unusually high reflectivity beyond $2 \mu$.

\section{SUMMARY}

Data are given on the reflecting power of monel metal, stellite, and zinc, in the visible spectrum and to $4 \mu$ in the infra-red spectrum. It was found that in the infra-red spectrum the reflectivity of monel metal is practically the same as that of nickel. In the visible spectrum the reflectivity is slightly lower than that of nickel.

A new determination was made of the reflecting power of a sample of stellite, the values obtained being about I per cent lower than previously observed in the visible spectrum.

An examination of the reflecting power of zinc shows that this metal is unique in having a deep depression in the reflectivity curve at $\mathrm{I} \mu$, followed by an unusually high reflectivity beyond $2 \mu$.

WASHINGTON, January 30, 1920. 